\title{
Clinical decisions and time since rest break: An analysis of
}

decision fatigue in nurses

Julia L. Allan ${ }^{1^{*}}$

Derek W. Johnston ${ }^{1}$

Daniel J. H. Powell1,2

Barbara Farquharson ${ }^{3}$

Martyn C. Jones ${ }^{4}$

George Leckie ${ }^{5}$

Marie Johnston ${ }^{1}$

1. Institute of Applied Health Sciences, University of Aberdeen, Aberdeen, UK.

2. Rowett Institute, University of Aberdeen, Aberdeen, UK

3. School of Nursing, Midwifery \& Social Care, Edinburgh Napier University, Edinburgh, UK.

4. School of Nursing \& Health Sciences, University of Dundee, Dundee, UK.

5. Centre for Multilevel Modelling, University of Bristol, Bristol, UK.

${ }^{*}$ Corresponding author

Dr Julia Allan

Health Psychology,

Institute of Applied Health Sciences,

Health Sciences Building,

School of Medicine, Medical Sciences \& Nutrition, University of Aberdeen, Foresterhill,

Aberdeen,

AB25 2ZD.

$+44(0) 1224438103$

j.allan@abdn.ac.uk

Acknowledgements: The parent study was funded by the Scottish Government's Chief Scientist Office (CZH/4/394).

Conflicts of Interest: None of the authors have conflicts of interest to declare. 
ABSTRACT

Objective: The present study investigates whether nurses working for a national medical telephone helpline show evidence of 'decision fatigue', as measured by a shift from effortful to easier and more conservative decisions as the time since their last rest break increases.

Methods: In an observational, repeated-measures study, data from 4,000 calls to 150 nurses working for the Scottish NHS 24 medical helpline (37\% of the national workforce) were modelled to determine whether the likelihood of a nurse deciding to refer a patient to another health professional the same day (the clinically safest, but most conservative and resource inefficient decision) varied according to the number of calls taken/time elapsed since a nurse's last rest break and/or since the start of shift. Analyses used mixedeffect logistic regression.

Results: For every consecutive call taken since last rest break, the odds of nurses making a conservative management decision (i.e. arranging for callers to see another health professional the same day), increased by $5.5 \%(p=.001$,

$6795 \% \mathrm{Cl}: 2.2 \%, 8.8 \%)$, an increase in odds of $20.5 \%$ per work hour $(\mathrm{p}<.001$, 95\% Cl: $9.1 \%, 33.2 \%$ ) or $49.0 \%$ (on average) from immediately after one break to immediately before the next. Decision making was not significantly 70 related to general or cumulative workload (calls or time elapsed since start of 71 shift). 
73 Conclusions: Every consecutive decision that nurses make since their last

74 break produces a predictable shift towards more conservative, and less

75 resource efficient decisions. Theoretical models of cognitive fatigue can

76 elucidate how and why this shift occurs, helping to identify potentially

77 modifiable determinants of patient care.

78

79 Keywords: decision making; fatigue; clinical decisions; nurses; efficiency

80

81

82 
INTRODUCTION

84

85
"We think, each of us, that we're much more rational than we are. And we think that we make our decisions because we have good reasons to make them." Daniel Kahneman (2012)

In order to make decisions about patients, health professionals must take in and weigh up relevant information and make a choice about the most appropriate course of action to take. While it is tempting to believe that the decisions of health professionals are based entirely on logic and a balanced weighing of the information available, human decision-making is susceptible to a wide range of individual, social and environmental influences. In a highlycited study of judicial decisions, Danziger, Levav and Avnaim-Pesso (2011) studied the parole decisions of court judges over the course of the working day. While the probability of a judge deciding to release a prisoner on parole at the beginning of the day was $\sim 65 \%$, this fell steadily to nearly $0 \%$ as time since the start of the court session wore on, returning to $\sim 65 \%$ after a food/rest break and again falling steadily to $0 \%$ towards the end of the day. The pattern reported fits with the psychological phenomenon 'decision fatigue': a state occurring when mental resources become depleted and/or when motivation to exert mental effort declines over time resulting in a measurable shift towards easier, safer, or more gratifying decisions and actions (Pignatiello, Martin \& Hickman, 2018). In the judge's case, the 'safest' 
and therefore mentally easiest option was to maintain the status quo (denying parole); the more depleted judges became, the more likely they were to select

110 this conservative option until an opportunity arose (in the form of a break) to

111 rest and replenish their resources. Since its publication, re-analyses of the

112 original data and data simulation studies have suggested that non-random

113 ordering of cases before judges and/or explicit planning of cases around

114 breaks is likely to explain some or all of the observed effects (Weinshall-

115 Margel \& Shapard, 2011; Glockner, 2016). However, the decision fatigue

116 phenomenon has been observed in multiple contexts where such factors are

117 less likely to be an issue.

118 Exertion-based depletion effects were first observed more than a 119 century ago with Arai (1912) noting in a study of continuous work over time 120 that "......continued work brings about a decrease in the efficiency of the 121 [mental] function exercised" (p. 114). Depletion effects are evident in a wide 122 range of contexts with people being, for example, more likely to cheat at 123 effortful tasks late in the day (Kouchaki \& Smith, 2014), more likely to give up 124 on diets at the end of the day (McKee, Ntoumani \& Taylor, 2014), more likely 125 to accept default options at the end of a series of consecutive decisions 126 (Levav, Heitmann, Hermann \& lyengar, 2010) and less able to perform well on 127 cognitive tests later in the day (Sievertsen, Gino \& Piovesan, 2016). Within 128 the healthcare context, compliance with hand hygiene protocols declines 129 predictably from the start to the end of shifts (Dai, Milkman, Hofmann \& 130 Staats, 2015; Chudleigh, Fletcher \& Gould, 2005), gastorenterologists are 131 more likely to detect polyps during morning than afternoon colonoscopies 132 (Chan, Cohen, \& Spiegel, 2009), and doctors prescribe more unnecessary 
133 134 Friedberg, Reyes Nieva, Birks, Meeker \& Fox, 2014). relevant consequences of that fatigue.

antibiotics towards the end than the start of clinic sessions (Linder, Doctor,

These decision fatigue effects have been primarily conceptualized as either the result of 'ego depletion', that is a decrease in the availability of the mental resources required to exert effortful self-control ( Baumeister, Pratlavsky, Muraven \& Tice, 1998; Baumeister, 2002), or of a progressive reduction in the motivation required to continue expending effort on the same task (Inzlicht \& Schmeichel, 2012; Inzlicht, Schmeichel \& Macrae, 2014;

Kurzban, Duckworth, Kable \& Myers, 2013). Recent registered replication reports (i.e. collections of independently conducted direct replications which follow an identical protocol) find no evidence of the former, self-control specific, ego-depletion effect (Hagger, Chatzisarantis, Alberts, Anggono, Batailler et al, 2016), but, as outlined above, many observational studies demonstrate predictable changes in behaviour and decision-making over time that are compatible with the motivational account (Danziger et al, 2011; Sievertsen et al, 2016; Dai et al, 2015). Within the context of nursing, research which directly contrasts these opposing accounts of fatigue finds motivational factors (perceived reward, perceived control) but not indicators of remaining resource (energy expenditure, work demands) to be consistently related to subjectively experienced fatigue over the working day (Johnston, Allan, Powell, Jones, Farquharson et al, 2018). However, this work looks only at the subjective fatigue reported by nurses and not at any potentially clinically

Both resource and motivational models would predict that decision fatigue should arise as a result of progressive increases in uninterrupted 'time 
on task' and consequently, that fatigue effects would be apparent in situations

159

where consecutive decisions are made without a break. Large numbers of consecutive decisions are made in frontline healthcare services where health professionals must make appropriate diagnostic and treatment decisions about a series of patients in turn. Consequently, any systematic changes in decision processes within this context would have clear implications for service provision (Pignatiello, Martin \& Hickman Jr, 2018). Furthermore, as the most important predictors of patient outcomes in any clinical context are likely to be fixed (e.g. severity of health condition), identifying new and potentially modifiable theoretical determinants of these outcomes is important. The present paper investigates the presence and extent of decision fatigue effects in the healthcare context, specifically testing whether the number of decisions made by health professionals since a break is, as predicted by theory, associated with a predictable shift towards more conservative (and therefore more expensive and less efficient) decisions. The study focuses on nurses working for the NHS 24 telephone helpline, an NHS Scotland wide service in which nurses assess callers' reported symptoms, make decisions about the most appropriate course of action to take, and direct callers onto primary or secondary care services where required. NHS 24 nurses deal with large numbers of cases and make many decisions each day. Importantly for the current research, unlike the judges in Danziger et al's study, nurses in the present study had no control over, or advance knowledge of, the content of incoming calls and limited control over the timing of their breaks, enabling a more robust test of the decision fatigue hypothesis. Using data from $\sim 4,000$ real clinical decisions made by 150 nurses, the likelihood of 
183 different decisions occurring is modelled from the beginning of a shift, up to

184 and following breaks during each shift. In line with theoretical accounts of

185 decision fatigue, it is expected that nurses will become increasingly likely to

186 make 'safer' and 'easier' (i.e. more conservative) treatment and management

187 decisions the more decisions they have made since their last break.

188

189 The present study tests two hypotheses. The decision fatigue hypothesis

190 predicts that nurses will become increasingly likely to make conservative

191 decisions the more uninterrupted time they have spent 'on task' within each

192 work shift. If this hypothesis is correct, nurses should make increasingly

193 conservative decisions the more consecutive decisions they have made

194 and/or the more time that has passed since their last break (or in the case of

195 the period prior to the first break of day, since they started their shift). A critical

196 prediction of this hypothesis is that any shift in decision making will occur as a

197 function of time/decisions since last break rather than time elapsed/decisions

198 made in total over the working day as breaks represent the theorised

199 opportunity to replenish motivational and/or cognitive resources. To confirm

200 that any effect detected relates specifically to time/decisions since break, a

201 second hypothesis -the general work hypothesis -is also tested to determine

202 whether decision making changes predictably over the working day as whole

203 irrespective of opportunities to replenish motivational and/or cognitive

204 resources (i.e. breaks). If this general hypothesis is correct, nurses should

205 become increasingly likely to make conservative decisions the more

206 consecutive decisions they have made and/or the more time that has passed 
in total since the start of their shift (regardless of breaks or time spent 'off-

208 task').

210 Each hypothesis is tested in our models with both number of decisions and

211 time elapsed as predictors. This results in four planned analyses. Specifically,

212 the likelihood that nurses would direct callers to another health professional

213 within 12 hours (the most conservative decision) is modelled in relation to (1)

214 the number of consecutive decisions made/calls taken since the last break (or

215 start of shift if prior to first break), (2) time since last break (or start of shift if

216 prior to first break), (3) decisions made/calls taken in total since the start of

217 shift regardless of breaks, and (4) total time spent at work since the start of 218 shift regardless of breaks.

\section{METHODS}

\section{Design and setting}

225 In a within-person, repeated measures study, data were collected on the

226 timing and number of calls and decisions made by nurses assessing callers to

227 the NHS 24 telephone service. NHS 24 is a nurse-led telephone (and now

228 online) advice service operating across Scotland and designed to provide $>5$

229 million members of the public with 24 hour access, 365 days a year, to health

230 services and information. Data for the present study were collected during a

231 comprehensive evaluation of the possible determinants and consequences of 
232 stress in nurses working for the NHS 24 service between 2008 and 2010. The

233 original parent study included physiological, behavioural and cognitive

234 measures collected in real time over two full working shifts in addition to

235 information about the calls received by nurses during the measurement

236 period. The study protocol (Allan, Farquharson, Choudhary, Johnston, Jones

237 \& Johnston, 2009) gives full details of these measures. Only methodological

238 information of relevance to the present study is presented here. The study

239 was reviewed and ethically approved by the NHS North of Scotland Research

240 Ethics Committee (05/S0801/136).

242 Participants and recruitment

243 All 465 nurses working in the four main NHS 24 call centres nationally or in

244 any of the 11 associated integrated local call centres were contacted with

245 study information and invited to take part in the study. Those interested in

246 participating $(n=171 / 37 \%$ of the national workforce) returned a signed

247 consent form and arranged suitable shifts for participation. Four participants

248 were excluded (as they were taking medication that would interfere with heart

249 rate recordings taken in the main study), two participants withdrew, it was not

250 possible to schedule data collection with thirteen further participants and data

251 was not available for an additional two participants. This resulted in a final

252 sample of 150 participants $(M$ age $=44$ years, $S D=7.5)$. Most participants

253 were female ( $n=142$, representative of the gender split in this workforce) and

254 were employed in seven different call centres across Scotland. Participants

255 worked an average of 22.6 hours per week $(S D=10.4)$, had been qualified for

256 an average of 21 years $(S D=8.3)$ and had been employed by NHS 24 for an 


\section{Procedure}

260 Participating nurses were asked to identify two 'typical' shifts from their next

261 month's rota where they would be available for participation and where they 262 would not be engaging in any non-standard activities (e.g. training, away days

263 etc). Data were collected on all calls received by each of the 150 participating

264 nurses over these two full working shifts. Call timing (time of day at which call

265 was taken) and duration (length of call in minutes/seconds) were

266 automatically recorded, and call outcomes (ambulance call-out, GP referral,

267 self-care, information provided, etc. see Table 1) were recorded by the nurse.

268 Most calls to the service are incoming (i.e. a standard call received from a

269 member of the public who has dialled the service or been routed from their

270 GPs number). However, during times of peak demand, urgent calls are

271 prioritised and less urgent calls are queued and returned by nurses later,

272 resulting in outgoing calls. Outgoing calls were excluded from the present

273 analysis as in these cases, nurses know in advance that the call is likely to be

274 non-urgent. Decision making within the service is supported by computerized

275 decision algorithms which highlight additional questions that nurses may wish

276 to ask /conditions that should be considered in response to particular

277 symptoms but in each case, the final decision on treatment / management is 278 made by an individual nurse.

\section{Data Coding}

281 Call outcomes - decisions taken by the nurse on which service the patient is 
referred to and with what level of urgency - were classified as either conservative (i.e. clinically safest and cognitively easier decisions) or not. All

284 decisions where the patient was advised to urgently seek, or was directly 285 referred to primary or secondary care within 12 hours via an urgent as 286 opposed to routine pathway were conceptualized as the conservative option: 287 in these cases, callers would be assessed by at least two health professionals 288 (the NHS 24 nurse and a primary/secondary care practitioner/first responder) 289 within the same day. As patients in these cases are retained within the 290 healthcare system and are assessed via phone and then again face to face 291 the same day, this was deemed the safest option clinically. Such decisions 292 are also cognitively 'easier' as nurses do not need to offer detailed information 293 or instructions to the patient, can resolve any uncertainty they feel about the 294 best course of action and can handover final responsibility for treatment and 295 management decisions to another health professional. These calls were 296 compared to all other calls where outcomes reflected less conservative 297 courses of action (where callers were not retained continuously in the 298 healthcare system but were advised to seek primary or secondary care 'within 29936 hours', or 'at their leisure').

301 Breaks from work were classified as any continuous period of 15 minutes or more between consecutive calls as this reflects the minimum standard break 303 length within the NHS 24 service. Outside of official breaks, nurses are 304 required to remain at their stations so that they are 'available for call' and 305 consequently during these times are unlikely to be truly 'off-task'. The position 306 of each call within the sequence of calls received since (i) start of shift; and (ii) 
the last break was computed as the predictor of interest.

\section{Analysis}

310 The final dataset had a two-level structure with triage decisions made during

311 incoming calls to the service (level-1; $k=3,948$ ) nested within individual nurses (level-2; $n=150)$. Nurses' decision making ( $1=$ conservative; $0=$ less conservative; as defined above) exhibited meaningful and significant

314 clustering within nurses $(\mathrm{ICC}=0.079, \mathrm{SE}=0.017, \mathrm{p}<.001)$ and were

315 therefore analysed using two-level mixed-effects logistic regression models.

316 All models included a random intercept, and a fixed effect of shift (first of the

317 two participation shifts $=0$; second shift $=1$ ). Since calls at certain times of 318 day may be more likely to involve serious conditions (warranting more urgent 319 responses), all analyses also included time of day (entered as a series of hour 320 of day dummy variables). As participating nurses worked in either one of the 321 larger, main call centres $(n=3)$ or in a smaller, regional call centre $(n=4)$, call 322 centre size was also controlled for in analyses.

324 To test hypothesis 1 (the decision fatigue hypothesis) two models were 325 estimated with the following fixed effects added: Model (i) number of decisions 326 i.e. calls since last break; and Model (ii) time elapsed in hours since the last 327 break. To test hypothesis 2 (the general work hypothesis) a further two 328 models were estimated, with the following fixed effects added: Model (iii) 329 number of decisions since the start of the shift; and Model (iv) time elapsed in 330 hours since the start of the shift. All analyses were carried out in Stata 15. 331 Statistical significance was set at $\alpha=.05$, corrected for multiple comparisons 
332 (Bonferroni corrected $\alpha=.0125$ ).

\section{RESULTS}

336 The participating nurses did not significantly differ from the rest of the NHS 24

337 workforce in terms of years qualified $\left(t_{(373)}=-0.817, p=0.41\right)$, years of 338 employment $(t(271)=-0.005, p=0.99)$, or number of hours worked per week $(t$ $339(430)=1.14, p=0.25)$.

$340 \quad$ During data collection, participating nurses dealt with 5,325 calls in

341 total (mean number of calls per nurse $=35.51 ; S D=11.72$ ). Of these, 95 calls

$342(1.8 \%)$ were coded as 'refused triage' meaning that either the call was

343 disconnected before a decision was made, or no decision could be agreed

344 between nurse and patient. As no decision was made during these 'refused

345 triage' calls, decisions in these cases were recorded as missing and were not

346 included in the analyses. A further 1,282 calls $(24.1 \%)$ were outgoing calls

347 and were also excluded from the analyses as nurses in these cases had

348 advance knowledge that the call content was non-urgent. The remaining

3493,948 calls were standard incoming calls and were included in the analyses.

350 During these incoming calls, 3,075 (77.9\%) of the decisions made were

351 classified as conservative and $873(22.1 \%)$ as not (see Table 1 for a

352 breakdown of call outcomes).

354 and involved an average of 13.78 incoming calls $(S D=6.82)$ each lasting an 355 average of 15.04 mins $(S D=6.08)$. There were 662 observed breaks lasting $\geq$ 35615 mins (mean $=2.30$ breaks per shift; $S D=1.47$. Median break length was 

25 minutes (IQR 19-35). The average time from the end of a break (or from the start of shift) to the end of the last call taken prior to the next break (or end of shift) was 2.14 hours $(S D=1.12)$. Nurses took an average of 4.35 incoming 360 calls $(S D=3.61)$ between breaks.

[Table 1 here]

364 Table 2 presents the fixed effect regression coefficients from all four models 365 where each model controls for time of day, shift (first or second), and size of 366 call centre. Model i (decisions since break) and ii (time since break) test the decision fatigue hypothesis: whether nurses make more conservative decisions the more consecutive decisions they have made and/or the more time that has

371 passed since their last break. The Model i results show that for every

372 additional call taken since their last break, the odds of nurses making a 373 conservative decision increased by $5.5 \%(p=.001,95 \% \mathrm{Cl}: 2.2 \%, 8.8 \%)$. The 374 results for Model ii show a similar effect with time elapsed. The odds of 375 nurses making a conservative decision increase by $20.5 \%$ for every hour that 376 passes since the last break $(p<.001,95 \% \mathrm{Cl}: 9.1 \%, 33.2 \%)$. This translates 377 to an increase in odds of $49.0 \%$ from the first call after one break to the final 378 call taken before the next break (i.e. an average of 2.14 hours later).

380 Models iii (decisions since start of shift) and iv (time since start of shift), test 381 the general work hypothesis: whether nurses simply become more likely to 
make more conservative decisions with more effort exerted during the whole shift. These models found no effect on decision making of number of calls (decisions) since the start of their shift $(p=.529)$ nor time elapsed since start of shift $(p=.766)$.

[Table 2 here]

\section{DISCUSSION}

The present analysis of $\sim 4,000$ clinical decisions made by nurses working for the telephone based NHS 24 service, revealed evidence of a predictable, mental-fatigue related bias in clinical decision making. Specifically, nurses made progressively more conservative and therefore more expensive and less efficient decisions as the time and the number of decisions made since their last rest break increased. For every call taken since last break, the odds of nurses recommending a treatment option that involved callers seeing another primary or secondary care professional within 12 hours increased by $5.5 \%$ : an increase in odds, on average, of $20.5 \%$ an hour or $49.0 \%$ from just after one break to just before the next. To convert this into relative probabilities: relative to the first person to call in after a nurse's break, the second caller will be $1.3 \%$ more likely to be retained within the healthcare system and seen the same day by another health professional, but the final caller before the next break will be $8.8 \%$ more likely to be seen the same day 
407 (i.e. a modelled probability of $75.4 \%$ immediately after a break, increasing to $40882.0 \%$ before the next break). This effect appears to be related specifically to

409 the number of decisions made / time that has elapsed since a nurse's last

410 break and not to an accumulation of general fatigue as decisions were

411 unaffected by the total number of calls taken or time at work over the shift as

412 a whole.

\section{Theoretical and clinical implications}

415 These results demonstrate that clinical decision makers are not immune to the

416 cognitive biases demonstrated in a range of other settings including law,

417 education and marketing (e.g. Danziger et al, 2011; Sievertsen et al, 2016;

418 Levav et al, 2010). Decision fatigue, i.e. the tendency to become increasingly

419 likely to go for the 'safe', 'default', or 'easy' option as the number of decisions

420 made increases, has long been exploited in the commercial sector. Economic

421 studies, for example, explicitly recommend that high mark-up options be

422 offered to customers late in a series of decisions to increase the likelihood of

423 them being accepted (Levav et al, 2010).

$424 \quad$ From a theoretical standpoint, the present findings cannot determine

425 whether the observed effects arise as the result of depletion of some general

426 cognitive resource or from a progressive shift in motivation away from current

427 task, but either way, they demonstrate that the effect is tied to the length of

428 continuous episodes of work (i.e. without a break) within a work period rather

429 than to the total or cumulative amount of work over the whole work period.

Within the clinical context, decision fatigue serves to bias decision

431 making in a conservative direction. This is optimal from a patient safety point 
432 of view as retaining patients within the healthcare system will maximize the

433 chances that any detectable health conditions are recognised and treated

434 quickly. However, an increased tendency to refer patients to primary or

435 secondary care services within a short time frame may also increase the

436 number of unnecessary investigations carried out, increase patient anxiety,

437 skew patient expectations of care, and make less efficient use of limited

438 resources. Referring large numbers of patients to primary and secondary care

439 undermines one of the core aims of medical telephone helplines. NHS 24 was

440 established by the Scottish Government in 2001 with a vision to ".....reduce

441 the ever increasing burden on existing services" (NHS 24, 2004). Our analysis

442 suggests that health professionals may become increasingly unable to fulfill

443 this vision as time since break increases. Furthermore, it implies that at times

444 when services are under the highest levels of demand, decision making may

445 be least efficient, since at these times, staff may be less likely to take the

446 breaks required to combat decision fatigue.

447 Importantly, our analysis suggests that it would not be necessary to

448 reduce total workload or shift length in order to reduce the effects of decision

449 fatigue in health professionals. Rather, it suggests that strategic scheduling of

450 (frequent, short) breaks would be the best way to ensure that decision making

451 remains efficient throughout shifts. This may be particularly relevant for those

452 who consciously recognise cognitive changes occurring as previous analyses

453 from the same dataset (Allan, Farquharson, Johnston, Jones, Choudhary \&

454 Johnston, 2014) indicate that nurses who report noticing that they are making

455 frequent cognitive failures (slips of attention and memory) are more likely in

456 general to refer callers on to other services. 
Much empirical data suggests that breaks from work are restorative, in

458 that they function to reduce, or even reverse, fatigue-related changes in

459 decision making and performance. A study of job performance over four

460 weeks (Binnewies, Sonnetag \& Mojza, 2010) found that when people had a

461 chance to fully recover from work demands during the weekend they reported

462 better performance of their core work tasks, were more likely to be proactive

463 and show initiative, and more likely to help others on their return to work.

464 Similarly, Dai et al (2015) demonstrated that while health professionals'

465 compliance with hand hygiene guidelines declined systematically over the

466 course of a 12-hour shift, workers with longer breaks between shifts showed

467 reduced depletion effects. While these studies look at 'breaks' in terms of

468 days off work, recent studies suggest that breaks in the day of 20-30 minutes

469 may be sufficient to completely remove cognitive depletion effects

470 (Sievertsen et al 2016). Similarly, there is some evidence to suggest that even

471 breaks of very short durations (3 minutes) are sufficient to mitigate depletion-

472 related declines in productivity (Dababneh, Swanson \& Shell, 2001).

$474 \quad$ Future Directions

475 Future research may be usefully focused on identifying optimal patterns of

476 breaks within the working shifts of healthcare professionals. Nursing shifts are

477 typically longer (8-12 hours) than in other professions (Stimpfel, Sloane \&

478 Aiken, 2012) and it is possible that multiple short breaks spaced across the

479 working shift (in addition to core meal and rest breaks) would improve rather

480 than reduce service efficiency. Sievertsen et al (2016) conclude in their study

481 of cognitive test performance over the day that the beneficial effect that 
resulted from a 20-30 minute break was larger than the initial depletion effect,

483 indicating that frequent breaks over the day may actually improve overall

484 performance. Future studies could investigate varying lengths of break, 485 possibly using an experimental design. Clearly, in a demanding and often 486 short staffed NHS setting, the logistical reality of providing staff with frequent 487 breaks is extremely challenging, but the present analysis suggests this is 488 worth exploring. It is estimated that primary care appointments cost the NHS an average of $£ 120$ each (NHS Information Services Division, 2012) so it is possible that frequent, short breaks, if they can safely reduce the number of

491 patients being referred onto other services, could be cost effective. For further development of theory, it is important to investigate whether 493 decision fatigue effects are indeed the result of progressive shifts in 494 motivation away from the current task. If so, then strategic incentives or 495 scheduled changes between different tasks may mitigate the effects. Finally, the present study focused on telephone based nurses who are 497 largely sedentary during working hours. Future studies should investigate 498 whether decision fatigue is enhanced or ameliorated in physically active, 499 ward-based nurses, that is, whether physical and mental fatigue effects 500 interact.

\section{Strengths and weaknesses}

503 The present study utilises existing data on the timing and outcome of $\sim 4,000$

504 real clinical decisions and the analytical approach allows important theoretical 505 predictions about changes in this clinical decision making to be tested within 506 people over time. As the data replicate the decision fatigue phenomenon 
507 observed in other professional groups (judges, GPs), it is likely that the results

508 are generalizable to other occupational settings. In terms of limitations, the

509 present study is observational in nature: it was not possible to experimentally

510 manipulate the timing and frequency of breaks. In addition, as data on call

511 content was not available, it was not possible to determine whether the

512 observed shift towards more conservative decisions was clinically less

513 'appropriate'. While similar studies indicate that clinicians' decision making

514 does become less appropriate over time (Linder et al, 2014), this could not be

515 tested directly in the present study. Finally, breaks were identified as any

516 period of $>15$ minutes between calls. While all such periods reflect a break

517 from active work, this method of classification is likely to include both officially

518 designated breaks where nurses are completely 'off-task' and periods of

519 inactivity at the workstation while still 'available for call'. The opportunities to

520 rest and recuperate from work may be reduced in the latter case and

521 consequently, the reported results may underestimate the magnitude of any

522 effect of breaks on decision making.

523

524 In conclusion, every consecutive decision that occurs, or hour that passes

525 since a break, produces a predictable and measurable change in nurses'

526 decision making that may function to gradually reduce service efficiency over

527 the working day. Future research should focus on identifying an optimal 528 pattern of breaks to minimize these effects. 


\section{1}

532 Allan, J.L., Farquharson, B., Choudhary, C.J., Johnston, D.W., Jones, M.C. \&

533 Johnston, M. (2009). Stress in telephone helpline nurses: research protocol

534 for a study of theoretical determinants, physiological aspects and behavioural

535 consequences. Journal of Advanced Nursing, 65, 2208-2215.

536

537 Allan, J.L., Farquharson, B., Johnston, D.W., Jones, M.C., Choudhary, C.J. \&

538 Johnston, M. (2014). Stress in telephone helpline nurses is associated with

539 failures of concentration, attention and memory, and with more conservative

540 referral decisions. British Journal of Health Psychology, 105, 200-213.

541

542 Arai, T. (1912). Mental Fatigue. New York City: Columbia University.

543

544

545 model of the self's executive function. Self Identity, 1, 129-136.

547 Baumeister, R.F., Bratslavsky, E., Muraven, M., \& Tice, D.M. (1998). Ego

548 depletion: Is the active self a limited resource? Journal of Personality and

549 Social Psychology, 74, 1252-1265.

550

551 Binnewies, C., Sonnetag, S., \& Mojza, E.J. (2010). Recovery during the

552 weekend and fluctuations in weekly job performance: A week-level study

553 examining intra-individual relationships. Journal of Occupational and

554 Organizational Psychology, 83, 419-441.

555 
556 Chan, M.Y., Cohen, H., \& Spiegel, M.R. (2009). Fewer polyps detected by

557 colonoscopy as the day progresses at a veteran's administration teaching

558 hospital. Clinical Gastroenterology and Hepatology, 7, 114-1142.

559

560 Chudleigh, J., Fletcher, M., \& Gould, D. (2005). Infection control in neonatal

561 intensive care units. Journal of Hospital Infection, 61, 123-129.

562

563 Dababneh, A.J., Swanson, N., \& Shell, R.L. (2001). Impact of added rest

564 breaks on the productivity and wellbeing of workers. Ergonomics, 44, 164-

565174.

566

567 Dai, H., Milkman, K.L., Hofmann, D.A., \& Staats, B.R. (2015). The impact of

568 time at work and time off from work on rule compliance: the case of hand

569 hygiene in health care. Journal of Applied Psychology, 100, 846-862.

570

571 Danziger, S., Levav, J., \& Avnaim-Pesso, L. (2011). Extraneous factors in 572 judicial decisions. Proceedings of the National Academy of Sciences, 108, $573 \quad 6889-6892$.

574

575 Glockner, A. (2016). The irrational hungry judge effect revisited: Simulations

576 reveal that the magnitude of the effect is overestimated. Judgment \& Decision

577 Making, 11, 601-610.

578 
579 Hagger, M.S., Chatzisarantis, N.L.D., Alberts, H., Anggono, C.O., Batailler, C.

580 et al. (2016). A multilab preregistered replication of the ego-depletion effect.

581 Perspectives on Psychological Science, 11, 546-573.

582

583 Inzlicht, M., \& Schmeichel, B.J. (2012). What is ego depletion? Toward a

584 mechanistic revision of the resource model of self control. Perspectives on

585 Psychological Science, 7, 450-463.

586

587 Inzlicht, M., Schmeichel, B.J., \& Macrae, C.N. (2014). Why self control seems

588 (but may not be) limited. Trends in Cognitive Science, 18, 127-133.

589

590 Johnston, D.W., Allan, J.L., Powell, D.J.H., Jones, M.C., Farquharson, B.,

591 Bell, C., \& Johnston, M. (2018). Why does work cause fatigue? A real-time

592 investigation of fatigue, and determinants of fatigue in nurses working 12-hour

593 shifts. Annals of Behavioral Medicine, Aug 16. doi: 10.1093/abm/kay065.

594 [Epub ahead of print].

595

596 Kahneman, D. (2012). Thinking fast and slow. London: Penguin Books.

597

598 Kouchaki, M., Smith, I.H. (2014). The morning morality effect: the influence of

599 time of day on unethical behavior. Psychological Science, 25, 95-102.

600

601 Kurzban, R., Duckworth, A., Kable, J.W., \& Myers, J. (2013). An opportunity

602 cost model of subjective effort and task performance. Behavioral and Brain

603 Sciences, 36, 10.1017/S0140525X12003196. 
605 Levav J, Heitmann M, Herrmann A, \& lyengar SS. Order in product

606 customization decisions: evidence from field experiments. J Polit Econ

$607 \quad 2010 ; 118: 274-299$.

608

609 Linder, J.A., Doctor, J.N., Friedberg, M.W., Reyes Nieva, H., Birks, C.,

610 Meeker, D., \& Fox, C.R. (2014). Time of day and the decision to prescribe

611 antibiotics. JAMA Internal Medicine, 174, 2029-2031.

612

613 McKee, H.C., Ntoumanis, N., \& Taylor, I.M. (2014). An ecological momentary

614 assessment of lapse occurrences in dieters. Annals of Behavioral Medicine,

$61548,300-310$.

616

617 NHS 24 Board Meeting Minutes, July 2004. Accessed from

618 http://www.nhs24.com/aboutus/nhs24board/agendasandpapers/2004/july/ /m

619 edia/nhs24/agendas\%20and\%20papers/2004/july/20040728\%20item\%2041

$620 \%$ 20blueprint\%20execution.ashx

621

622 NHS Scotland Information Services Division. (2012). Cost Book. NHS

623 Scotland.

624

625 Pignatiello, G.A., Martin, R.J., \& Hickman Jr, R.L. (2018). Decision fatigue: A

626 conceptual analysis. Journal of Health Psychology, doi:

627 10.1177/1359105318763510. [Epub ahead of print]. 
629 Sievertsen, H.H., Gino, F., \& Piovesan, M. (2016). Cognitive fatigue

630 influences students' performance on standardized tests. Proceedings of the

631 National Academy of Sciences, 113, 2621-2624.

632

633 Stimpfel, A.W., Sloane, D.M., \& Aiken, L.H. The longer the shifts for hospital

634 nurses, the higher the levels of burnout and patient dissatisfaction. Health

635 Affairs, 31, 2501-2509.

636

637 Weinshall-Margel, K., \& Shapard, J. (2011). Overlooked factors in the analysis

638 of parole decisions. Proceedings of the National Academy of Sciences, 108, 639 E833. 
Decision taken by nurse

Frequency (n)

Referral within 12 hours (conservative) - Total 3075

Ambulance sent 390

Urgently attend accident \& emergency 277

Urgently attend GP surgery 2382

Urgently speak to GP 17

Othera

Referral outside 12 hour window (less conservative)- Total

Attend GP surgery

Speak to GP

Information provided

Self-care advised

Other

Refused Triage

Total calls handled emergency centre

${ }^{b}$ Other less conservative referrals included to pharmacist, dentist, midwife, health visitor, service. 
651 Table 2. Mixed effects logistic regressions predicting likelihood of nurses

652 making conservative decisions from (i) number of decisions (calls) since last break, (ii) hours since last break, (iii) number of decisions (calls) since start of shift and (iv) hours since start of shift. Time was entered as a series of dummy 655 hour of day variables and is included in all analyses shown. Complete tables 656 (showing time variables in full) can be found in Supplementary Table S1.

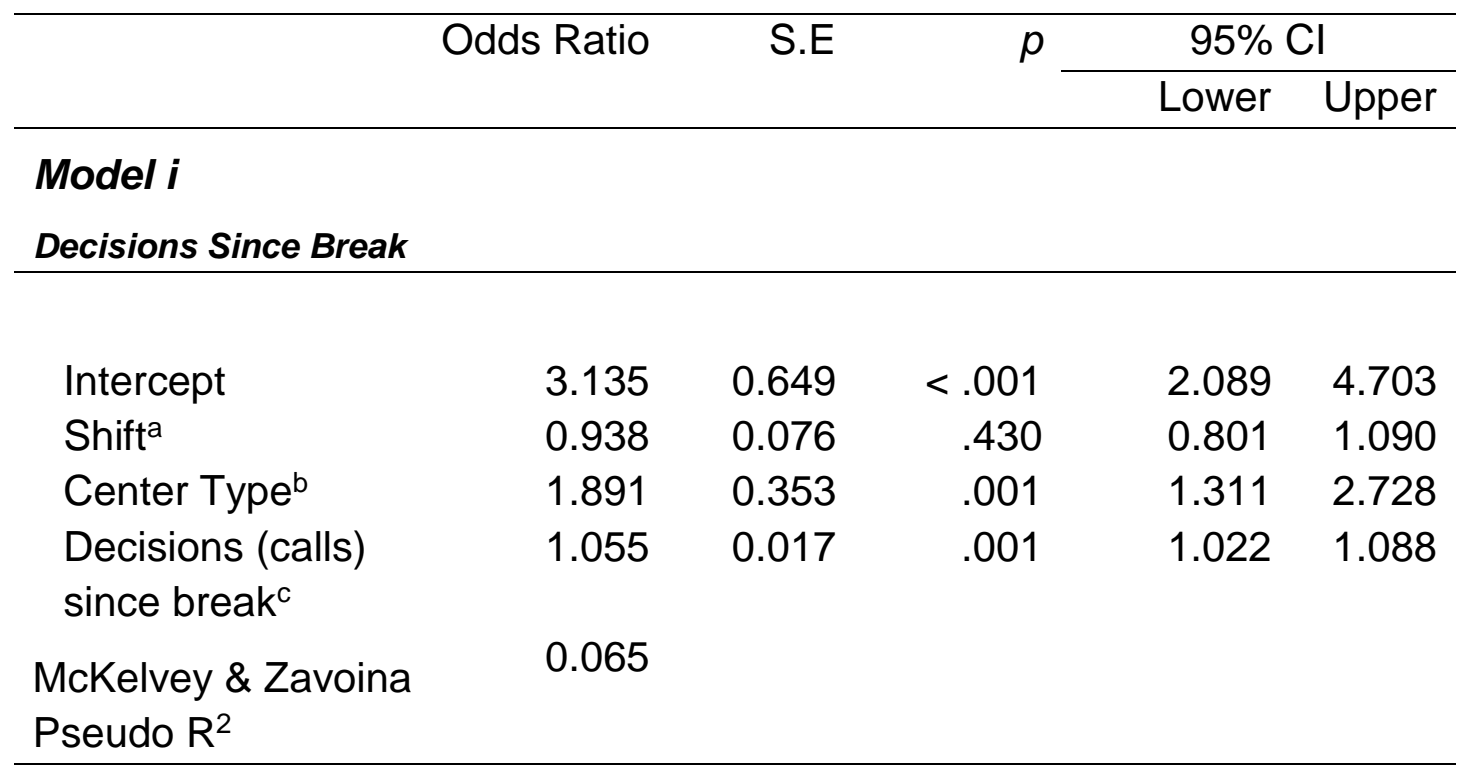

\section{Model ii}

Time Since Break

$\begin{array}{lrrrrr}\text { Intercept } & 3.067 & 0.635 & <.001 & 2.044 & 4.603 \\ \text { Shifta } & 0.938 & 0.0766 & .425 & 0.800 & 1.098 \\ \text { Center Type } & 1.825 & 0.344 & <.001 & 1.289 & 2.668 \\ \text { Time (hrs) since } & 1.205 & 0.061 & <.001 & 1.091 & 1.332\end{array}$

break $^{c}$

McKelvey \& Zavoina

0.066

Pseudo $\mathrm{R}^{2}$

\section{Model iii}

\section{Decisions Since Starting}

$\begin{array}{lrrrrr}\text { Intercept } & 3.410 & 0.739 & <.001 & 2.230 & 5.215 \\ \text { Shift }^{\mathrm{a}} & 0.933 & 0.753 & .390 & 0.796 & 1.093 \\ \text { Center Type } & 1.836 & 0.344 & .001 & 1.272 & 2.651\end{array}$


Decisions (calls)

$1.006 \quad 0.009$

.529

0.988

1.023

since start of shift

McKelvey \& Zavoina $\quad 0.060$

Pseudo $\mathrm{R}^{2}$

\section{Model iv}

\section{Time Since Starting}

\begin{tabular}{lrrrrr} 
Intercept & 3.685 & 0.841 & $<.001$ & 2.356 & 5.765 \\
Shift $^{\mathrm{a}}$ & 0.936 & 0.076 & .412 & 0.799 & 1.097 \\
Center Type & 1.830 & 0.343 & $<.001$ & 1.267 & 2.643 \\
Time (hrs) since start & 0.992 & 0.026 & .766 & 0.942 & 1.045 \\
of shift & & & & & \\
McKelvey \& Zavoina & 0.060 & & & & \\
Pseudo R & & & & & \\
\hline
\end{tabular}

658

659

a Shift coded as $0=$ shift one, $1=$ shift two; ${ }^{b}$ center type coded as $0=$ three large centers, $1=$ 660 four small centers; ${ }^{c}$ Since last break, or start of shift if in first call period.

661 